\title{
Die Veränderung von selbsteingeschätzter Beratungskompetenz und Selbstwirksamkeitserwartung: Eine Interventionsstudie bei Lehramtsstudierenden
}

\author{
Anne Schlosser, Jennifer Paetsch, Daniela Sauer \\ Otto-Friedrich-Universität Bamberg
}

Zusammenfassung: Lehramtsstudierende fühlen sich auf ihre spätere Beratungsaufgabe oft nur wenig vorbereitet, obgleich diese im schulischen Kontext zum grundlegenden Aufgabenbereich von Lehrkräften gehört. Dies legt eine Förderung der Beratungskompetenz in der ersten Phase der Lehramtsausbildung nahe. Im vorliegenden Beitrag wird untersucht, inwiefern die selbsteingeschätzte Beratungskompetenz und die beratungsbezogene Selbstwirksamkeitserwartung von Lehramtsstudierenden durch theoretische und praktische Lerngelegenheiten verändert werden können. Es wurde eine Interventionsstudie (Prä-Post-Vergleich) mit Kontrollgruppendesign durchgeführt $(\mathrm{N}=292)$. Die Ergebnisse zeigen im Bereich der selbsteingeschätzten Kompetenzen signifikante Verbesserungen mit sehr hohen Effektstärken. Hinsichtlich der Selbstwirksamkeit kann ebenfalls eine positive Veränderung mit mittlerer Effektstärke durch die Intervention verzeichnet werden. Eine Veränderung der Selbstwirksamkeit ließ sich dabei nur durch den praktischen und nicht durch den theoretischen Interventionsteil feststellen. In der Kontrollgruppe wurden keine vergleichbaren Veränderungen beobachtet.

Schlüsselbegriffe: Beratungskompetenz von Lehrkräften, peer-education, Selbstwirksamkeit, selbsteingeschätzte Kompetenzen, Praxiserfahrung

\section{Changes in self-assessment of counseling skills and self-efficacy: an intervention study with pre-service teachers}

Summary: Many pre-service teachers feel insufficiently prepared for counseling tasks in their professional routine. This, however, is expected a basic task of a teacher in all school types. Therefore, initial teacher education should promote counseling skills. This intervention study examines changes in self-estimated counseling skills and anticipated counseling self-efficacy during theoretical and practical parts of a teacher training program. We investigated 292 pre-service teachers using prepost-control-group-design. Results reveal positive changes with high effect sizes for self-estimated counseling skills and with medium effect sizes for self-efficacy. Furthermore, we only found for the practical part of the intervention positive effects for self-efficacy; there was no increase during the theoretical training component. No changes in the control group were observed.

Keywords: teacher counseling competences, Peer Education, self-efficacy, self-estimated competences, practical experience

Beratungsgespräche mit Schülerinnen und Schülern sowie deren Eltern zählen zu den grundlegenden professionellen Aufgaben (KMK, 2004) sowie zu den entscheidenden Gesprächstypen im Berufsalltag von Lehrpersonen (Gartmeier, 2018; Sauer, 2015). Dem- entsprechend gilt Beratungskompetenz als wichtige Facette professioneller Handlungskompetenz von Lehrkräften, der im Wandel von Schule und Unterricht eine zunehmende Bedeutung zugesprochen wird (vgl. Baumert \& Kunter, 2013; Kunina-Habenicht et al., 2012; 
Schnebel \& Jürgens, 2012; Wild \& Lütje-Klose, 2017). Viele bereits ausgebildete Lehrkräfte schätzen ihre Beratungskompetenzen jedoch als eher gering ein; sie fühlen sich nicht ausreichend für die Übernahme von Beratungsaufgaben in der Praxis qualifiziert und erleben Elterngespräche als belastend (Dusolt, 2008; Freyaldenhoven, 2005; Hertel, 2009; Hertel \& Schmitz, 2010; Rothland, 2013; Wild, 2003; Wildt, 2004).

Angebote zur Förderung von Beratungsund Gesprächsführungskompetenzen sind in der universitären Lehrkräftebildung eher selten implementiert (vgl. Hertel, 2009; Strasser \& Gruber, 2003; Wild, 2003), in den letzten Jahren sind jedoch verstärkt Bemühungen in diese Richtung zu verzeichnen (vgl. Kunter \& Pohlmann, 2015; Drechsel, et al., 2020). So liegen bereits empirisch überprüfte (relativ zeitaufwendige) Lehrkonzepte vor (vgl. Hertel, 2009; Aich, 2011), die eine Erlernbarkeit von Beratungskompetenz bereits belegen konnten (vgl. Aich, 2015; Gartmeier, 2018; Gerich, 2017; Gerich, Trittel \& Schmitz, 2017). Weiterhin zeigen Untersuchungen, dass eine Steigerung der Beratungskompetenz mit der domänenspezifischen Selbstwirksamkeitserwartung und den Selbsteinschätzungen assoziiert sind (z. B. Kücholl, Lazarides, Westphal \& Lohse-Bossenz, 2018) und dass Lehrkräfte, die sich im Führen von Elterngesprächen weniger kompetent einschätzen, auch seltener Elterngespräche anbieten (Wild, 2003; Hertel, 2017).

Im Rahmen der Förderung von handlungsbezogenen Beratungskompetenzen bei Lehrkräften kommen theoretische Einführungen und praktische Übungen zum Einsatz (z. B. Hertel, 2009). Über die genauen Wirkmechanismen von theoretischen und praktischen Interventionsanteilen ist jedoch wenig bekannt (z. B. Lubitz, 2007; Hertel, 2009). Der vorliegende Beitrag knüpft an diesem Forschungsdesiderat an und untersucht Veränderungen der selbsteingeschätzten Kompetenzen und der Selbstwirksamkeit von Lehramtsstudierenden zum Thema schulische Beratung durch den Besuch einer Qualifizierungsmaßnahme. Ein be- sonderer Fokus liegt dabei auf der Frage, inwieweit theoretische und praktische Ausbildungsanteile zur Verbesserung der selbsteingeschätzten Beratungskompetenzen und zur Stärkung der beratungsbezogenen Selbstwirksamkeit beitragen. Die Teilnehmenden besuchten im Rahmen eines Seminars „Beratung im schulischen Kontext (Peer-Beratungstraining) " sieben dozierendengeleitete theoretische Seminarsitzungen und absolvierten in einem sich anschließenden praktischen Training verschiedene Übungen zur Gesprächsführung in schulbezogenen Beratungsgesprächen, welche von Peer-Edukatorinnen und Edukatoren angeleitet wurden (für eine ausführliche Beschreibung der Qualifizierungsmaßnahme siehe Drechsel et al., 2020).

\section{Theoretischer Hintergrund}

\section{Beratungskompetenz in der Lehrkräftebildung}

In der pädagogisch-psychologischen Literatur werden unterschiedliche Definitionen von pädagogischer Beratung beschrieben (zur Übersicht z. B. Hertel, 2009). Übereinstimmend wird dabei eine Abgrenzung zwischen dem alltäglichen Beratungsverständnis und einem professionellen Begriff von Beratung vorgenommen (z. B. Grewe, 2005). Professionelle Beratung wird dabei weniger im Sinne eines direktiven Beratungsverständnisses konzeptioniert, vielmehr wird betont, dass es sich um einen freiwilligen Prozess handelt, der bei Adressatinnen und Adressaten Selbstklärungsprozesse anregt und Ressourcen aktiviert (Nußbeck, 2006). Die Beratung in der Schule umfasst zahlreiche Themen- und Problembereiche, zusätzlich sind Lehrkräfte gefordert, nicht nur über Wissen zu verschiedenen Inhaltsbereichen zu verfügen (z. B. Lernprobleme), sondern auch über geeignete Beratungs- bzw. Gesprächsführungsstrategien (vgl. Bruder, Hertel, Gerich \& Schmitz, 2014). Strasser und Gruber (2003) definieren die Beratungskompetenz als „[...] fachliches Wissen um Sachverhalte und um die Wirksamkeit von Maßnahmen, [welches] [...] auf der 
Grundlage personaler Ressourcen reflektierte Erfahrung [erlaubt], die befähigt, Wissen situationsangemessen und effektiv anzuwenden, was zu beraterischem Erfolg, also dem Erreichen der im Beratungsprozess gesetzten Ziele, führt “ (S. 388).

Im Bereich der Beratungskompetenz von Lehrkräften liegen einige Studien vor, die eine theoretisch begründete Kompetenzmodellierung mit empirischer Überprüfung vornehmen (u. a. Bruder, Hertel \& Schmitz, 2010; Gartmeier, 2018; Gerich, 2017; Hertel, 2009). Basierend auf Modellen von Hertel (2009) und Bruder (2011) wurde von Gerich, Trittel und Schmitz (2017) ein Modell mit vier Kompetenzdimensionen extrahiert und durch eine faktoranalytische Untersuchung bestätigt: Kommunikationsskills, Diagnostikskills, Problemlöseskills, Bewältigungsskills. Das Modell der Gesprächsführungskompetenz wurde von Gartmeier (2018) für den medizinischen und schulischen Bereich entwickelt und umfasst die Kompetenzfacetten Interpersonale Beziehung, Kooperative Problemlösung und Strukturierung des Elterngesprächs.

Zusammenfassend zeigt sich, dass zwar verschiedene Modelle vorliegen, diese jedoch große Gemeinsamkeiten aufweisen. Grundlage bilden beratungswissenschaftliche Ansätze, die für die Lehrkräftebildung angepasst wurden und den besonderen Stellenwert von Beziehungsgestaltung und Gesprächsführung hervorheben. Zudem sind die postulierten und überprüften Kompetenzfacetten prozess- und handlungsorientiert ausgerichtet. Wissen über Beratung und Aspekte des Selbstkonzeptes werden hier als Voraussetzungen des professionellen Beratungshandelns konzeptualisiert (vgl. Gartmeier, 2018; Gerich, 2017; Hertel, 2009).

\section{Prädiktoren und Fördermöglichkeiten von Beratungskompetenzen in der Lehrkräftebildung}

Einige der Studien, die eine Kompetenzmodellierung der Beratungskompetenzen vornehmen, beziehen auch mögliche Prädiktoren in ihre Untersuchung ein (z. B. Gartmeier, 2018;
Gerich, 2017; Hertel, 2009). So zeigt die Studie von Bruder (2011) anhand einer Stichprobe mit praktizierenden Gymnasiallehrkräften signifikant positive Zusammenhänge zwischen Selbstwirksamkeit und handlungsbezogener Beratungskompetenz (ermittelt mit einem Fallszenariotest), respektive zwischen Selbstwirksamkeit und den Teilkompetenzen Ressourcen- und Lösungsorientierung sowie Pädagogischem Wissen und Diagnostizieren. Zudem erwies sich in der Studie von Bruder und Kollegen (2010) die beratungsbezogene Selbstwirksamkeitserwartung als ein signifikanter Prädiktor der Selbsteinschätzung von Beratungskompetenzen.

Insgesamt weisen die vorliegenden Befunde darauf hin, dass beratungs- und lernstrategiebezogenes Wissen und v. a. Selbstwirksamkeitserwartung positiv mit Beratungskompetenz von ausgebildeten Lehrkräften assoziiert sind (z. B. Bruder, 2011; Bruder et al., 2010; Hertel, 2009). Unklar ist bislang, wie diese Erkenntnisse genutzt werden können, um bereits in der universitären Lehrkräfteausbildung Beratungskompetenz von Lehramtsstudierenden sowie deren Selbstwirksamkeit zu fördern.

Die vorliegenden Kompetenzmodelle und Befunde legen für die Gestaltung von universitären Lerngelegenheiten nahe, Studierende zunächst mit den Anforderungen von Beratungsaufgaben vertraut zu machen und die Entwicklung funktionaler Überzeugungen zum Thema Beratung zu unterstützen (vgl. Sauer, 2017). Es wird davon ausgegangen, dass Lehramtsstudierende ihr erworbenes Wissen als anschlussfähig betrachten, wenn die eigene Selbstwirksamkeit in Beratungssituationen positiv bewertet wird und Motivation sowie Interesse zum Weiterlernen am Thema geweckt wurden (Hertel, 2009). Gartmeier und Kollegen (2015) verdeutlichen die Notwendigkeit von anschlussfähigem Wissen zur Kompetenzentwicklung. Sie zeigten, dass die Kommunikationsfähigkeiten von Studierenden des Lehramts und der Medizin durch eine kombinierte Form von E-Learning und Rollenspiel mit Video-Feedback am effektivsten gesteigert werden konn- 
ten. Relevant scheint dabei zu sein, dass für die Lernenden eine nachvollziehbare Verknüpfung zwischen Praxis und Theorie besteht (Korthagen, 2010; Renkl, 2014). Qualifizierungen für (angehende) Lehrkräfte setzen verstärkt an der beratungsbezogenen Wissensvermittlung an, wobei häufig praktische Übungen, im Sinne reduzierter Praxiserfahrungen (vgl. Gartmeier, 2018; Hertel, 2009) und Anregungen zur Reflexion im Fokus stehen (bspw. Furr \& Carroll, 2003; Jennings, Goh, Skovholt, Hanson \& Banerjee-Stevens, 2003). In Interventionsstudien haben sich die Maßnahmen mit praktischen Anteilen als wirksam zur Verbesserung beratungsbezogener Kompetenzen von Lehramtsstudierenden erwiesen (z. B. Dotger, Dotger \& Maher, 2010; Dotger, Harris \& Hansel, 2008; Aich, 2011; Gartmeier, 2018; Gerich, 2017). Als wirksame Methoden werden hierbei Lernen am Modell, gezieltes Feedback, intensive Praxisanleitungen, Supervision, Reflexion sowie systematisches Training an Teilaufgaben betrachtet (z. B. Ball \& Forzani, 2009; Strasser \& Gruber, 2003; Watts \& Lawson, 2009). Empirische Untersuchungen bestätigen dabei, dass v. a. Feedback (z. B. Gerich, Trittel \& Schmitz, 2017; Lambert et al., 2002; Mc Leod, 2013; Strasser \& Gruber, 2003) sowie Selbstreflexion (z.B. Rønnestad \& Skovholt, 2003; Thiel, 2003; Strasser \& Gruber, 2003) wirksam für die Förderung der Beratungskompetenzen sind. Tang, Addison, LaSure-Bryant, Norman, O'Connell und Stewart-Sicking (2004) konnten zudem nachweisen, dass die Steigerung der beratungsbezogenen Selbstwirksamkeit abhängig von der Dauer der Praktika und anderer Berufserfahrungen ist.

In der Interventionsstudie von Hertel (2009) zeigten sich bei Studierenden geringere Effekte hinsichtlich der personalen Ressourcen und des Beratungswissens als bei bereits ausgebildeten Lehrkräften. Begründet wird diese Diskrepanz mit der fehlenden Beratungserfahrung, was die Überwachung eigener Kompetenzen erschweren soll. Insgesamt verweisen vorliegende Forschungsbefunde auf die prinzipielle Wirksamkeit von beratungsbezogenen Inter- ventionen bei Lehramtsstudierenden sowie auf die Relevanz praktischer Einheiten für die Verbesserung von Beratungskompetenz, wobei die Nutzbarkeit der theoretischen Inhalte durch die Aneignung anschlussfähigen Wissens für die folgende Praxisphase von besonderer Relevanz ist.

\section{Die Rolle von Selbstwirksamkeit in der Lehrkräfteausbildung}

Die Lehrkräfte-Selbstwirksamkeitserwartung ist eine zentrale Komponente professioneller Kompetenz von Lehrkräften (Baumert \& Kunter, 2006). Selbstwirksamkeitserwartungen wirken sich positiv auf das Lehrkräfteverhalten im Unterricht aus (z. B. Schipper, Goei, de Vries \& van Veen, 2018; Summers, Davis \& Woolfolk Hoy, 2017; Zee \& Koomen, 2016 für eine Übersicht). Auch für die beratungsbezogene Selbstwirksamkeitserwartung konnte ein $\mathrm{Zu}-$ sammenhang mit professionellem Beratungshandeln von Lehrkräften nachgewiesen werden (z. B. Hertel, 2009). Um bereits im Studium Beratungskompetenz von angehenden Lehrkräften zu fördern, scheint die Steigerung der Selbstwirksamkeitserwartung deshalb ein geeigneter Ansatzpunkt zu sein (vgl. Hertel, 2009). Wie die allgemeine Selbstwirksamkeitsforschung gezeigt hat, spielt die Überzeugung über eigenes Können eine bedeutende Rolle auf der bereichsübergreifenden Handlungsebene (Schwarzer \& Jerusalem, 2010). Definiert wird die Selbstwirksamkeitserwartung nach Bandura (1986):

Perceived self-efficacy is defined as peoples judgment's of their capabilities to organize and execute courses of action required to attain designated types of performances. It is concerned not with the skills one has but with judgments of what one can do with whatever skills one possesses.

Im deutschsprachigen Raum erweitern Schwarzer und Jerusalem (2010) diese: es handelt sich um die Bewältigung neuer oder schwieriger Situationen, welche Handlungen voraussetzen, die von der Gewohnheit abweichen und eine 
gewisse Investition im Sinne von Anstrengung und Ausdauer verlangen. Unterschieden wird die allgemeine Selbstwirksamkeit von einer domänenspezifischen Selbstwirksamkeit (Bandura, 1986). Im Folgenden wird sich auf die domänenspezifische, respektive die beratungsbezogene Selbstwirksamkeitserwartung bezogen.

Die zwei bedeutendsten Einflussfaktoren für Selbstwirksamkeitserwartung sind direkte persönliche Erfahrungen, die aufgrund ihrer Authentizität den größten Einfluss haben, sowie stellvertretende Erfahrungen, die eine gute Möglichkeit bieten, eigene Fähigkeiten in Relation zu den Modellen einzuschätzen. Durch direkte persönliche Erfahrungen wird die Wirksamkeit des eigenen Handelns und der eigenen Kompetenz in Problemlösesituationen sichtbar. Erfolgreiche Erfahrungen fördern dabei die Selbstwirksamkeitserwartung, während Misserfolge diese schwächen. Bezogen auf universitäre Lerngelegenheiten bedeutet dies, dass v. a. praktische Lerngelegenheiten einen positiven Einfluss auf die Selbstwirksamkeitserfahrung haben können. Stellvertretende Erfahrungen dagegen können auch in theoretischen Lerneinheiten gemacht werden, da hier durch Modelllernen Wissen und Strategien zur Problembewältigung vermittelt werden (Bandura, 1986). Aus der Selbstwirksamkeitsforschung ist bekannt, dass die eigene Wirksamkeitsüberzeugung einen entscheidenden Einfluss auf den Lernprozess in der Schule oder der Universität hat (Bandura, 2007; Lent, Larkin \& Brown, 1986; Pajares \& Kranzler, 1995; Siegel, Galassi \& Ware, 1985; Zimmerman \& Kitsantas, 1997; Zimmerman, 2000). Relevant scheint die eigene Wirksamkeitsüberzeugung für Lehramtsstudierende zum einen zu sein, da diese positiv mit einer hohen Erwartung an die eigenen Kompetenzen, mit einem besseren Arbeitszeitmanagement, anhaltenderer Ausdauer, dem frühzeitigen Herausfinden von falschen bzw. unwirksamen Strategien und einer akkurateren Arbeitsweise korreliert. Zum anderen setzen sich Lernende, die sich als hoch selbstwirksam wahrnehmen, höhere Ziele und haben höhere Erwartungen an sich, außerdem zeigen sie mehr
Flexibilität bei der Lösungssuche, evaluieren eigene Ergebnisse gewissenhafter und erreichen höhere intellektuelle Leistungen (Bandura, 2007; Zimmerman, 2000). Besonders im Bereich der intrinsischen Motivation spielt die Selbstwirksamkeit eine besondere Rolle, da es relevant ist, ob Studierende an ihre akademische Fähigkeit glauben oder nicht (Zimmerman, 2000). Dabei hat die Einschätzung der eigenen Kompetenzen nach Zimmerman (2000) einen Einfluss auf die Hauptindikatoren von akademischer Motivation - Wahl der Aktivität, Anstrengung und Ausdauer sowie emotionale Reaktionen (vgl. Kücholl, Westphal, Lazarides \& Gronostaj, 2019).

Eine hohe Selbstwirksamkeitserwartung ist demnach förderlich für den Lernprozess und kann im Studium den Erwerb von Wissen und Kompetenzen erleichtern sowie deren Qualität erhöhen. Selbstwirksamkeitserwartung scheint demzufolge als geeigneter Ansatzpunkt in der beratungsbezogenen Ausbildung von Lehramtsstudierenden. Obwohl nahezu immer darauf hingewiesen wird, dass sich Selbstwirksamkeitserwartung nur domänenspezifisch bestimmen lässt, existieren bisher kaum Studien, die spezifisch auf die beratungsbezogene LehrkräfteSelbstwirksamkeit eingehen (Baumert \& Kunter, 2006; Tschannen-Morane, Woolfolk Hoy $\&$ Hoy, 1998). Vorliegende empirische Studien zeigen, dass es hinsichtlich der allgemeinen Lehrkräfte-Selbstwirksamkeit zu einer Steigerung während des Studiums und einer Regression bei den ersten Praxiserfahrungen im Berufseinstieg kommt (Tschannen-Morane et al., 1998; Woolfok Hoy \& Burke Spero, 2005). Offen bleibt die Frage, welche hochschuldidaktischen Maßnahmen im Studium zu einer Steigerung der Selbstwirksamkeit führen können. Die hierzu vorliegenden empirischen Befunde sind nicht eindeutig. Eine Überprüfung der Selbstwirksamkeit über das Studium allgemein wurde u. a. von Schulte, Bögeholz und Watermann (2008) durchgeführt, hierbei konnte anhand des Vergleichs von Studierenden zu Beginn und am Ende des Studiums ein allgemeiner signifikanter Anstieg der Selbstwirksamkeit im 
Studium hinsichtlich der Bereiche Unterrichten, Leistungsbeurteilung und auch der allgemeinen Selbstwirksamkeit gezeigt werden. Unbekannt ist, welche Studieninhalte zur Förderung der Selbstwirksamkeitserwartung beitrugen. In der Längsschnittstudie von Schüle, Besa, Schriek und Arnold (2017) wurde ein Rückgang der individuellen Lehrkräfteselbstwirksamkeit während der ersten drei Fachsemester und eine signifikante Steigerung nach der ersten Praxiseinheit im vierten Semester beobachtet. Dagegen konnten Weß, Priemer, Weusmann, Ludwig, Sorge und Neumann (2020) in einer Querschnittsstudie keine Unterschiede in der Selbstwirksamkeitserwartung hinsichtlich Planung, Durchführung und Reflexion eigener Instruktionen zwischen Studierenden zu Beginn und am Ende des Studiums feststellen. Woolfolk Hoy und Burke Spero (2005) sowie Swan, Wolf und Cano (2011) zeigen einen signifikanten Anstieg der Selbstwirksamkeitserwartung der Lehramtsstudierenden durch intensive und authentische Praxisphasen. Auch außeruniversitäre pädagogische Erfahrungen haben sich als bedeutsam für Selbstwirksamkeitserwartungen für das Beraten von Schülerinnen und Schülern erwiesen (Kücholl, Lazarides \& Westphal, 2020).

Dass Selbstwirksamkeitserwartung auch durch theoretische Studieninhalte gesteigert werden kann, zeigen die Ergebnisse von Mergler und Tangen (2010). Des Weiteren gibt es empirische Evidenzen für die Wirksamkeit von hybriden Lehrformaten, die sowohl aus theoretischen als auch aus praktischen Lehrformaten bestehen (z. B. Lubitz, 2007). In einer beratungsbezogenen Trainingsstudie, mit praktischen und theoretischen Anteilen, mit Lehramtsstudierenden konnte Hertel (2009) hinsichtlich des wahrgenommenen Kompetenzzuwachses und der Selbstwirksamkeit signifikante Unterschiede nach der Intervention im Vergleich zu einer Kontrollgruppe zeigen. Unklar bleibt hier und auch in den zuvor angeführten Studien, inwieweit die praktischen bzw. theoretischen Anteile auf die Selbstwirksamkeit bzw. den wahrgenommenen Kompetenzzuwachs wirken.
Einige Autorinnen und Autoren betonen angesichts ihrer Befunde jedoch explizit die Relevanz von praktischen Erfahrungen zur Steigerung der Selbstwirksamkeit (vgl. Hertel, 2009; Lubitz, 2007; Schüle et al., 2017).

\section{Selbsteingeschätzte Kompetenz und Selbstwirksamkeitserwartung}

In der empirischen Forschung existieren zahlreiche Studien, die relevante Kompetenzen angehender Lehrkräfte über Selbsteinschätzungen erfassen (z. B. Braun \& Hannover, 2009; König, Kaiser \& Felbrich, 2012). Dabei scheinen Instrumente zur Selbsteinschätzung verstärkt Aspekte der Performanz (Können bzw. Handeln) zu fokussieren und weniger des Wissens (vgl. König et al., 2012). Daran anknüpfend wird die Selbsteinschätzung der Kompetenzen in theoretischen und empirischen Auseinandersetzungen nicht mehr als rein kognitives Konstrukt verstanden, sondern vielmehr als ein Konstrukt, welches durch motivationale und selbstregulative Aspekte sowie Überzeugungen und Professionswissen mehrdimensional beschrieben werden kann (Baumert \& Kunter, 2006; Suhlmann, 1986; Klingebiel, Mähler \& Kuhn, 2020). Weiterhin weisen vorliegende Befunde darauf hin, dass die Ausprägung von Selbsteinschätzungen, stärker als Wissenstestungen, mit Merkmalen wie z. B. Selbstwirksamkeitserwartungen, Selbstkonzepten oder Maßen des Selbstvertrauens zusammenhängen (vgl. König et al., 2012).

Selbstwirksamkeit und selbsteingeschätzte Kompetenz sind demzufolge zwei Konzepte, die stark miteinander assoziiert sind (z. B. Bach, 2013; Cramer, 2010; Meyer, 2014) und sich wechselseitig beeinflussen. Gemeinsam ist den beiden Konstrukten zudem der stark subjektive Charakter und die Notwendigkeit der domänenspezifischen Beschreibung.

Auch in dem theoretischen Modell zur Entwicklung der Selbstwirksamkeit von Lehrkräften nach Tschannen-Morane und Kollegen (1998) spielen Kompetenzselbsteinschätzungen eine zentrale Rolle. Ausgehend von diesem Modell wird deutlich, dass die Wirksamkeitsüber- 
zeugung das Resultat einer Analyse der Anforderungssituation in Kombination mit der Bewertung der eigenen Kompetenzen ist, welche zur Situationsbewältigung notwendig erscheinen. Selbstwirksamkeitserwartung beeinflusst wiederum eigene Zielsetzungen sowie Anstrengung und Ausdauer, welche in einer spezifischen Anforderungssituation aufgewendet werden (Tschannen-Morane et al., 1998). Übertragen auf Beratungskompetenz illustriert das Modell die Rolle der subjektiven Bewertung der eigenen Fähigkeiten: In Kombination mit der subjektiven Beurteilung der Anforderungen von Beratungssituationen soll sich demnach die Selbstwirksamkeitsüberzeugung als Beraterin oder Berater entwickeln. Schlussfolgernd hat die selbsteingeschätzte Beratungskompetenz einen direkten Einfluss auf die Selbstwirksamkeitsüberzeugung, umgekehrt wirkt diese, vermittelt über das Handlungsergebnis, ebenfalls auf die Selbsteinschätzung der Beratungskompetenz. Der Zusammenhang zwischen selbsteingeschätzter Beratungskompetenz und Selbstwirksamkeit wurde für Lehramtsstudierende bereits gezeigt (Kücholl, Lazarides, Westphal \& LohseBossenz, 2018).

Zusammenfassend lässt sich feststellen, dass Selbstwirksamkeit und selbsteingeschätzte Kompetenz zwei Konstrukte sind, die nur schwer voneinander abzugrenzen sind und die sich gegenseitig beeinflussen. Selbstwirksamkeitserwartung umfasst jedoch neben der Beschreibung der eigenen Kompetenzen auch das Vertrauen in diese in Problemlösesituationen und ist somit auf konkrete Anforderungen bezogen, während die Selbsteinschätzung der Kompetenzen nicht situationsspezifisch sein muss (Tschannen-Morane et al., 1998).

\section{Fragestellung und Hypothesen}

Vorliegende Studien zeigen, dass Selbstwirksamkeit und selbsteingeschätzte Kompetenz von Lehramtsstudierenden durch Interventionen und praktische Erfahrungen gefördert werden können (vgl. Hertel, 2009; Lubitz, 2007; Schulte et al., 2008; Braun \& Hannover, 2008;
König et al., 2012); auch speziell für die domänenspezifische beratungsbezogene Selbstwirksamkeit und Selbsteinschätzung von Kompetenzen konnte dies gezeigt werden (z. B. Bruder, 2011; Hertel, 2009; Gerich, Trittel \& Schmitz, 2017). Unklar ist jedoch, wie die theoretischen und praktischen Anteile der Qualifizierungsmaßnahmen sich jeweils auf die Entwicklung der Selbstwirksamkeit und der Selbsteinschätzungen auswirken. Die Frage, welche Rolle theoretische Erfahrungen der Studierenden auf der einen und praktische Erfahrungen auf der anderen Seite bzw. theoretische und praktische Erfahrungen in Kombination spielen, ist bislang noch nicht explizit empirisch untersucht worden. Theoriebasiert wird davon ausgegangen, dass praktische Erfahrungen einen größeren Einfluss auf die Entwicklung der Selbstwirksamkeitsüberzeugung und selbsteingeschätzten handlungsbezogenen Kompetenzen haben als theoretischer Input (vgl. Tschannen-Morane et al., 1998; Schwarzer \& Jerusalem, 2010). Hier knüpft der vorliegende Beitrag an und untersucht domänenspezifisch, inwieweit sich Selbstwirksamkeit und die selbsteingeschätzte Kompetenz zum Thema schulische Beratung von Lehramtsstudierenden durch theoretischen Input (Wissensvermittlung) und praktische Übungen, im Sinne reduzierter Praxiserfahrungen (bspw. Lernen am Modell, Rollenspiele), verändern. Es wurde eine Interventionsstudie (Prä-PostVergleich) mit Kontrollgruppendesign durchgeführt, um folgende Hypothesen zu untersuchen:

Hypothese 1. Studierende, die an einem Training mit theoretischen und praktischen Anteilen zur Förderung der schulischen Beratungskompetenz teilgenommen haben, zeigen einen höheren Zuwachs hinsichtlich (a) der beratungsbezogenen Selbstwirksamkeitserwartung und (b) der selbsteingeschätzten Beratungskompetenz als Studierende der Kontrollgruppe, die an keiner Intervention teilgenommen haben.

Hypothese $2 a$. Eine Steigerung der beratungsbezogenen Selbstwirksamkeitserwartung der Interventionsteilnehmenden lässt sich erst nach 
dem praktischen Teil der Qualifizierungsmaßnahme feststellen.

Hypothese $2 b$. Die Selbsteinschätzung der Beratungskompetenzen (in Bezug auf Wissen und Können) der Interventionsteilnehmenden erhöht sich sowohl nach dem theoretischen Teil als auch (nochmal) nach dem Besuch des praktischen Teils der Qualifizierungsmaßnahme.

\section{Methode}

\section{Untersuchungsdesign und Intervention}

Für die vorliegende Untersuchung wurden Daten verwendet, die im Rahmen des Projektes „Beratung im schulischen Kontext" (im Rahmen der Qualitätsoffensive Lehrerbildung) an der Otto-FriedrichUniversität Bamberg erhoben wurden und Teil einer umfassenderen Evaluationsmaßnahme sind (vgl. Drechsel et al., 2020). Die Erhebung erfolgte über einen Zeitraum von drei Semestern (2017-2019), wobei ein Peer-Beratungstraining (d.h. ein Seminar) pro Semester angeboten wurde. Es wurde ein PräPost-Design mit drei Messzeitpunkten und Kontrollgruppe gewählt. Der Prätest erfolgte vor Besuch des Peer-Beratungstrainings, der Posttest 1 nach Besuch des theoretischen Teils und der Posttest 2 nach Besuch des praktischen Teils der Qualifizierung. Eine Kontrollgruppe wurde zu zwei Zeitpunkten befragt, wobei der Abstand zwischen Prä- und Posttest dem zwischen Prä- und Posttest 2 der Interventionsgruppe entspricht. Studierende der Kontrollgruppe besuchten keine Veranstaltungen zum Thema Beratung und nahmen an einer Online-Befragung teil. Die Intervention besteht aus theoretischer Wissensvermittlung (sieben Sitzungen) und praktischen Übungen (1,5-tägiges Wochenendseminar). Die grundlegende Konzeptionierung des Peer-Beratungstrainings erfolgte auf Basis humanistischer und systemischer Beratungsansätze (vgl. Drechsel et al., 2020). Der Theorieteil umfasste dabei einen dozierendengeleiteten Überblick zum Thema Beratung im Anwendungsfeld Schule, wobei unter anderem grundlegende Definitionen von Beratung und Beratungskompetenz (u. a. Sauer, 2017; Strasser \& Gruber, 2003), Besonderheiten der schulischen Beratung (Schnebel \& Jürgens, 2012), verschiedene Beratungsansätze (u. a. Rogers, 1951; Schlippe \& Schweitzer, 2010), Funktionen und Fehlformen (Schwarzer \& Posse, 2005), Beratungsanlässe (Hertel, 2009) sowie
Zielformulierungen (Fuller \& Taylor, 2012) zum Thema Beratung im schulischen Kontext thematisiert wurden. Im praktischen Teil wurden nach einer kurzen Phase der Vorwissensaktivierung unter Leitung von Peer-Edukatorinnen und -Edukatoren in zufällig zugeteilten Kleingruppen (acht bis zwölf Teilnehmende) systemische Fragen sowie Problemexploration und Schwierigkeiten, die in Beratungsgesprächen im schulischen Kontext aufkommen können, in Rollenspielen beobachtet sowie in Dreiergruppen mit rotierenden Rollen (Berater/in, Ratsuchender/ Ratsuchende, Beobachter/in) angewendet. Die Reflexion wurde durch gezielte Beobachtungsaufträge (z. B. „Wie gelingt es der Lehrkraft, die Problemsicht der ratsuchenden Mutter zu erkunden? Welche systemischen Fragen fallen dir auf?") angeregt und in der Kleingruppe sowie im Plenum diskutiert. Es wurden also reduzierte und angeleitete Praxisphasen angeboten, in denen direkte persönliche Erfahrungen gemacht werden konnten. Peer-Edukatorinnen und -Edukatoren sind Lehramtsstudierende mit einem Beratungsschwerpunkt in ihrem Studium, also Studierende des Beratungslehramtes bzw. der Schulpsychologie. Diese Studiengänge zeichnen sich durch mehr pädagogisch-psychologische Ausbildungsinhalte und Qualifizierung in Beratung im schulischen Kontext aus. Die Peer-Edukatorinnen und -Edukatoren absolvierten vorab eine Qualifizierung und erhielten ein ausführliches Manual zur Anleitung des Beratungstrainings (vgl. Drechsel et al., 2020).

\section{Stichprobe}

Insgesamt nahmen 292 Lehramtsstudierende (davon $88,4 \%$ Frauen) über drei Studiensemester an der Studie teil, davon gehören 102 Studierende der Kontrollgruppe und 190 der Interventionsgruppe an. An der Intervention in Form eines Seminars (Wahloption im Pflichtcurriculum) haben im Wintersemester 2017/18 59, im Sommersemester 2018 63 und im Wintersemester 2018/19 68 Lehramtsstudierende teilgenommen, während nur in den ersten beiden Semestern Teilnehmende für die Kontrollgruppe (Onlinebefragung) akquiriert wurden (Wintersemester 2017/18: 13; Sommersemester 2018: 89). In der Interventionsgruppe haben 185 Lehramtsstudierende zum ersten Messzeitpunkt an der Befragung teilgenommen, 177 zum zweiten und 166 zum dritten. Diese unterschiedlichen Stichprobengrößen können durch Fehlzeiten der Studierenden erklärt werden, wobei nur zehn Teilnehmende die Intervention nach dem ersten Messzeitpunkt 
abgebrochen haben (unsystematischer Dropout ${ }^{1}$ $N=10)$. Das Alter zum Zeitpunkt des Prätestes lag im Durchschnitt bei $M=21.86(S D=3.47)$. Für die Zahl der im Lehramt studierten Semester ergab sich ein signifikanter Unterschied $(T(267)=-2.69$, $\mathrm{p}=.008)$ zwischen den Teilnehmenden der Kontrollgruppe $(M=4.50 ; S D=3.73)$ und den Teilnehmenden der Interventionsgruppe $(M=3.4 ; S D=3.10)$. In der Interventions- (IG) und Kontrollgruppe (KG) nahmen die Studierenden des Grundschullehramts (IG: 61\%; KG: 44,6\%) den mit Abstand größten Anteil ein, gefolgt von den Studierenden für die Lehrämter der Mittel- und Realschule (IG: 22\%; KG: 16,3\%) und Gymnasium (IG: 11,6\%; KG: $14,1 \%$ ) und der Beruflichen Bildung (IG: 5,8\%; KG $13 \%)$, zudem nahmen in der Kontrollgruppe $12 \%$ aus dem Masterstudiengang Bildungsmanagement/ Schulbildung teil.

\section{Instrumente}

\section{Selbsteingeschätzte Kompetenzen}

Zur Erfassung der selbsteingeschätzten Kompetenzen zur schulischen Beratung wurde ein Instrument entwickelt und in einer Pilotierungsstudie erprobt (vgl. Drechsel et al., 2020, S. 206-209). Die Skala zur Erfassung der selbsteingeschätzten Kompetenzen (SK) besteht aus 48 Items, welche selbsteingeschätztes Beratungswissen (26 Items, bspw. Ich weiß, wie man ein Beratungsgespräch mit einem Ratsuchenden strukturiert) und selbsteingeschätztes Können (22 Items, bspw. Ich kann ein Beratungsgespräch für den Ratsuchenden gut nachvollziehbar strukturieren) abdeckt (achtstufige Antwortskala von trifft überhaupt nicht $z u$ bis trifft sehr $z u$ ). Inhaltlich umfasst die Skala, angelehnt an die Konzeptionierungen der Beratungskompetenz von Hertel (2009), Bruder (2011) und Gerich (2017), Items zu Beziehungsgestaltung (z. B. Ich kann meine Aufmerksamkeit dem Ratsuchenden gegenüber zum Ausdruck bringen), Gesprächstechniken (z. B. Ich weiß, wie man Aussagen des Ratsuchenden paraphrasiert), Umgang mit Herausforderungen (z. B. Ich weiß, wie man mit einem sehr kritischen Elternteil in einem Beratungsgespräch

Es wurden keine systematischen Unterschiede hinsichtlich demografischer Daten, Selbstwirksamkeit und selbsteingeschätzter Kompetenz zwischen den Teilnehmenden der gesamten Intervention und den Teilnehmenden, die an beiden Posttestungen nicht teilgenommen haben, gefunden umgeht), selbstregulative Fähigkeiten (z. B. Ich kann Ratsuchende an andere Beratungsinstanzen verweisen, wenn ein Fall meine Grenzen als beratende Lehrkraft übertritt; vgl. Drechsel et al., 2020). Die Items spiegeln dabei Inhalte der theoretischen Sitzungen und praktischen Übungen wider (siehe oben, vgl. Drechsel et al., 2020). Die faktoranalytische Untersuchung (Hauptkomponentenanalyse mit Varimax-Rotation) zeigte zu beiden Messzeitpunkten die Extraktion von nur einem Faktor und bestätigt somit die Eindimensionalität der Skala (Prätest: Eigenwert 24.88, $51,83 \%$ erklärte Varianz; Posttest 2: Eigenwert 34.99, $72,89 \%$ erklärte Varianz). Die interne Konsistenz der Skala ist sehr hoch (Prätest: $N=253, \alpha=.98$; Posttest 1: $N=152, \alpha=.97$; Posttest 2: $N=253, \alpha=.99$ ).

\section{Selbstwirksamkeit}

Zur Erfassung der Selbstwirksamkeit wurde ein Instrument aus der Studie von Hertel (2009) eingesetzt. Die Skala besteht aus neun Items, ist auf einer sechsstufigen Skala von trifft überhaupt nicht $z u$ bis trifft voll und ganz $z u$ einzuschätzen und zeigt eine gute Reliabilität (Prätest: $N=255, \alpha=.84$; Posttest 1 : $N=170, \alpha=.85$; Posttest 2: $N=244, \alpha=.88)$. Beispielitem: Möglichen Schwierigkeiten im Elterngespräch sehe ich gelassen entgegen, weil ich meinen Fähigkeiten vertrauen kann. Inhaltlich wurden drei Aspekte der Selbstwirksamkeitserwartung überprüft: Selbstwirksamkeit bezüglich Beratungsgesprächen, emotionale Grundhaltung gegenüber Beratungsgesprächen und Sicherheit im Umgang mit Eltern.

\section{Analysestrategie}

Die statistische Auswertung wurde mit dem Statistikprogramm Statistical Package for the Social Sciences (SPSS) in Version 25 durchgeführt. Zunächst wurden mit t-Tests für unabhängige Stichproben die Unterschiede zwischen Interventions- und Kontrollgruppe zum ersten Messzeitpunkt überprüft. Die Veränderung der selbsteingeschätzten Kompetenzen und der Selbstwirksamkeit innerhalb der Interventionsgruppe wurde anhand einer einfaktoriellen Varianzanalyse mit Messwiederholung zum ersten und dritten Messzeitpunkt untersucht. Die Überprüfung der Gruppenunterschiede nach der Intervention ( $\mathrm{Hy}-$ pothese 1, Wirksamkeit der Intervention) erfolgte anhand einer multiplen linearen Regression, die es ermöglicht, den Kompetenzzuwachs durch mehrere Prädiktoren vorherzusagen (vgl. Cohen, Cohen, West \& Aiken, 2003). Als abhängige Variable wurde 
der Posttest 2 (dritter Messzeitpunkt), als unabhängige Variablen der Prätest (erster Messzeitpunkt), die Gruppenzugehörigkeit ( 1 = Interventionsgruppe, $0=$ Kontrollgruppe) und die Anzahl der Lehramtssemester verwendet. Zur Überprüfung von Hypothese $2 a$ und $2 b$ (Effekte des theoretischen Teils und des praktischen Teils) wurden einfaktorielle Varianzanalysen mit Messwiederholung in der Interventionsgruppe durchgeführt. Allen Berechnungen lag ein Signifikanzniveau von $\mathrm{p}<.05$ zugrunde.

\section{Ergebnisse}

\section{Deskriptive Ergebnisse}

Es bestehen keine signifikanten Unterschiede zwischen den Studierenden der unterschiedlichen Semester bzw. Trainings in Hinblick auf die untersuchten Variablen, daher wird die Interventionsgruppe als eine Gruppe betrachtet. In Tabelle 1 werden die deskriptiven Werte für alle Messzeitpunkte angegeben. Im Mittel schätzten alle Studierenden ihre eigenen beratungsbezogenen Kompetenzen zum ersten Messzeitpunkt gering ein: Die Einschätzungen der Kontrollgruppe: $M=2.38, S D=1.45$ und die der Interventionsgruppe: $M=2.20, S D=1.34$ liegen im unteren Bereich der 8-stufigen Selbsteinschätzungsskala $(\mathrm{Min}=0, \mathrm{Max}=7)$. Die Überprüfung der Gruppenunterschiede vor der Intervention zeigte keine signifikanten Unterschiede zwischen Kontroll- und Interventionsgruppe $(T(251)=0.97, p=.331)$. Anhand der
Mittelwerte der selbsteingeschätzten Kompetenzen ist in der Interventionsgruppe zu allen Messzeitpunkten deskriptiv eine Steigerung zu beobachten. Zum letzten Messzeitpunkt schätzten die Studierenden der Interventionsgruppe ihre Kompetenzen im Mittel hoch ein $(M=5.83$, $S D=0.69)$. Zudem zeigte eine stetige Verringerung der Standardabweichungen in der Interventionsgruppe, dass die Einschätzung durch die Interventionsteilnahme homogener wird. Die durchschnittliche Einschätzung der Kontrollgruppe blieb zum letzten Messzeitpunkt weiterhin gering $(M=2.89, S D=1.71)$.

Die Selbstwirksamkeitserwartung wurde in der Interventions- und in der Kontrollgruppe zum ersten Messzeitpunkt bereits relativ hoch eingeschätzt, beide Mittelwerte befinden sich im oberen Bereich (Kontrollgruppe: $M=3.77$, $S D=0.77$; Interventionsgruppe: $M=3.92$, $S D=0.74)$ der sechsstufigen Skala $(\mathrm{Min}=1$, Max =6). Die Überprüfung der Gruppenunterschiede zum ersten Messzeitpunkt zeigte auch hier keine signifikanten Unterschiede zwischen den Gruppen $(T(153)=1.15, p=.133)$. Die Mittelwerte der Kontrollgruppe sind zu beiden Messzeitpunkten nahezu identisch. Auch die Mittelwerte der Interventionsgruppe zu den ersten beiden Messzeitpunkten unterscheiden sich kaum, eine Steigerung ist zum letzten Messzeitpunkt erkennbar $(M=4.20, S D=0.69)$.

Die bivariaten Zusammenhänge zwischen den verschiedenen Variablen (Kontroll- und

Tab. 1: Deskriptive Statistik der Interventions- und Kontrollgruppe

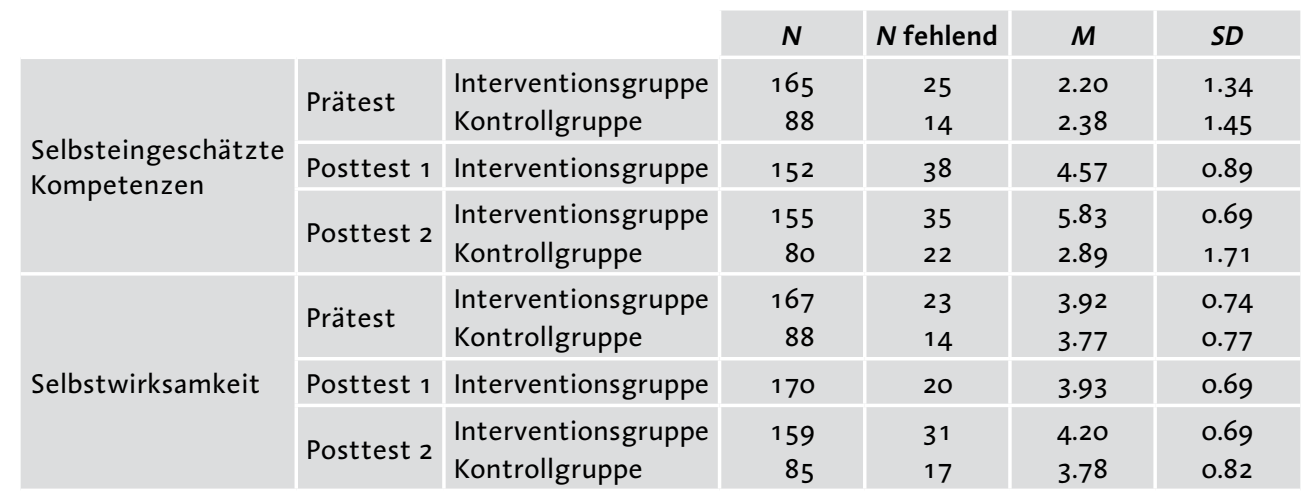


Tab. 2: Bivariate Korrelationen der untersuchten Konstrukte

(1) Selbsteingeschätzte Kompetenz (Prätest)

(2) Selbsteingeschätzte Kompetenz (Posttest 1)

(3) Selbsteingeschätzte Kompetenz (Posttest 2)

(4) Selbstwirksamkeitserwartung (Prätest)

(5) Selbstwirksamkeitserwartung (Posttest 1)

(6) Selbstwirksamkeitserwartung (Posttest 2)
(1)

(2)

(3)

(4)

(5)

$.29^{\star *}$
$.20^{\star *}$
$.23^{\star \star}$
$.22^{\star \star}$
.07

Anmerkungen: * signifikant auf Signifikanzniveau .01 (zweiseitig); ** signifikant auf Signifikanzniveau .05 (zweiseitig).

Interventionsgruppe wurden zusammengefasst) sind Tabelle 2 zu entnehmen. Die Variablen sind zu nahezu allen Messzeitpunkten hoch signifikant miteinander korreliert. Sowohl die Selbstwirksamkeitserwartung als auch die selbsteingeschätzten Kompetenzen verschiedener Messzeitpunkte sind signifikant korreliert. Darüber hinaus bestehen signifikante Zusammenhänge zwischen Selbstwirksamkeitserwartung und selbsteingeschätzten Kompetenzen zu gleichen Messzeitpunkten. Im Folgenden werden die Hypothesen inferenzstatistisch geprüft.

\section{Veränderungen durch die Intervention: Vergleich von Kontrollgruppe und Interventionsgruppe}

Zunächst wurde untersucht, wie sich die selbsteingeschätzten Kompetenzen und die Selbstwirksamkeit in der Interventionsgruppe vom ersten zum dritten Messzeitpunkt verändern. Die Ergebnisse der Varianzanalysen mit Messwiederholung zeigten in der Interventions- gruppe erwartungsgemäß signifikante Verbesserungen vom ersten zum dritten Messzeitpunkt für die selbsteingeschätzten Kompetenzen $\left(F(1,136)=901.09, p<.001\right.$, partielles $\left.\eta^{2}=.869\right)$ und die Selbstwirksamkeitsskala $(F(1,143)=$ $36.19, p<.001$, partielles $\eta^{2}=.202$ ), mit hohen Effektstärken. In beiden Fällen konnte demzufolge eine signifikante Steigerung der selbsteingeschätzten Kompetenz sowie der Selbstwirksamkeit in der Interventionsgruppe festgestellt werden.

Ein Vergleich der Gruppenunterschiede zur Prüfung der Interventionseffekte (Hypothese 1) erfolgte anhand von multiplen linearen Regressionen unter Einbezug der Prätestergebnisse (vgl. Cohen et al., 2003). Als Kontrollvariable wurde die Anzahl der studierten Lehramtssemester berücksichtigt, da sich Studierende der Interventions- und Kontrollgruppe hierin signifikant unterschieden.

Hinsichtlich der Selbstwirksamkeitserwartung erwiesen sich die Prätesteinschätzung $(\beta=.66, p<.001)$ und die Gruppenzugehörig-

Tab. 3: Regressionsanalysen zur Prüfung der Interventionseffekte

\begin{tabular}{|c|c|c|c|c|c|c|c|c|}
\hline \multirow{3}{*}{ Prädiktor } & \multicolumn{8}{|c|}{ Kriteriumsvariable (Posttest 2) } \\
\hline & \multicolumn{4}{|c|}{$\begin{array}{c}\text { selbsteingeschätzte Kompetenz } \\
\text { (Post) }\end{array}$} & \multicolumn{4}{|c|}{$\begin{array}{l}\text { Selbstwirksamkeit } \\
\text { (Post) }\end{array}$} \\
\hline & $b$ & $S E$ & $\beta$ & $p$ & $b$ & $S E$ & $\beta$ & $p$ \\
\hline Prätest & .36 & .05 & .28 & $<.001$ & .67 & .05 & .66 & $<.001$ \\
\hline Gruppenzugehörigkeit & 3.00 & .15 & .83 & $<.001$ & .40 & .08 & .26 & $<.001$ \\
\hline Anzahl der Lehramtssemester & .04 & .03 & .07 & .119 & -.02 & .01 & -.06 & .213 \\
\hline
\end{tabular}

Anmerkungen: $\beta$ =standardisierte Regressionskoeffizienten; Gruppenzugehörigkeit: 0 = Kontrollgruppe, 1 = Interventionsgruppe 
keit $(\beta=.26, p<.001)$ als signifikante Prädiktoren der Posttesteinschätzung (vgl. Tabelle 3 ). Hypothese 1a kann angenommen werden: Die Selbstwirksamkeitserwartung der Interventionsteilnehmenden ist signifikant höher als die der Kontrollgruppe unter Kontrolle der Prätestergebnisse. Die Ergebnisse im Bereich der selbsteingeschätzten Kompetenzen zeigten ein ähnliches Bild. Sowohl die Einschätzungen im Prätest $(\beta=.28, p<.001)$ als auch die Interventionsteilnahme $(\beta=.83, p<.001)$ haben einen signifikant positiven Effekt auf die Einschätzung der eigenen Kompetenzen im Posttest. Die Anzahl der Lehramtssemester ist hingegen kein signifikanter Prädiktor (vgl. Tabelle 3). Hypothese $1 \mathrm{~b}$ wird bestätigt.

\section{Veränderungen innerhalb der Interventionsgruppe nach Theorie- und Praxisteil}

Die Überprüfung der Veränderungen in der Interventionsgruppe unter Berücksichtigung aller drei Messzeitpunkte (getrennte Betrachtung von Theorie- und Praxisteil) erfolgte mithilfe von einfaktoriellen Varianzanalysen mit Messwiederholung. Vermutet wurde, dass sich die Selbstwirksamkeitserwartung insbesondere nach dem praktischen Teil der Intervention erhöht und nicht nach dem theoretischen Teil (Hypothese $2 \mathrm{a}$ ).

Für die Selbstwirksamkeitserwartung zeigte sich ein signifikanter Haupteffekt $(F(2,276)=$ $25.80, p<.001$, partielles $\eta^{2}=0.157$ ). Post-hocTests zeigten, dass signifikante Veränderungen nur vom ersten zum letzten Messzeitpunkt (Mittlere Differenz: -.31, 95\% - CI[(-.43) $(-.18)], p<.001)$ und vom zweiten zum letzten Messzeitpunkt (Mittlere Differenz: -.31, 95\% - CI[(-.42) - (-.20)], $p<.001)$ mit einer mittleren Effektstärke feststellbar sind. Vom ersten zum zweiten Messzeitpunkt (hier fand der Theorieteil statt) veränderte sich die Selbstwirksamkeitserwartung dahingehend nicht signifikant $(p=1)$. Hypothese 2a kann also angenommen werden, da ein signifikanter Unterschied der Selbstwirksamkeitserwartung nach dem Praxis- teil festgestellt werden konnte, wohingegen keine Veränderung nach dem Theorieteil gezeigt wurde.

Weiterhin wurde vermutet, dass eine Veränderung hinsichtlich der selbsteingeschätzten Kompetenzen durch den praktischen und den theoretischen Teil der Intervention erzielt werden kann (Hypothese 2b). Die Überprüfung der Unterschiede mittels Varianzanalyse ergab einen signifikanten Haupteffekt $(F(2,234)=$ $567.02, p<.001$, partielles $\eta^{2}=.83$ ). Dabei zeigten Post-Hoc-Tests signifikante Unterschiede bezüglich der gesamten Intervention (Mittlere Differenz: $-3.62,95 \%$ - CI [(-3.93) - (-3.31)], $p<.001)$, aber auch im Vergleich der ersten beiden Messzeitpunkte (Theorieteil - Mittlere Differenz: $-2.52,95 \%$ - CI $[(-2.81)-(-2.22)]$, $p<.001)$ sowie im Vergleich der letzten beiden Messzeitpunkte (Praxisteil - Mittlere Differenz: $-1.10,95 \%-$ CI $[(-1.28)-(-.92)], p<.001)$. Hypothese $2 \mathrm{~b}$ kann angenommen werden, da alle Messzeitpunktvergleiche signifikante Veränderungen zeigen.

\section{Diskussion}

Beratung im schulischen Kontext ist unumstritten eine wichtige Aufgabe von Lehrkräften, die jedoch in deren Ausbildung bislang nur wenig systematisch berücksichtigt wurde (vgl. Kunter \& Pohlmann, 2015). In jüngster Zeit werden verstärkt regional Qualifikationsmaßnahmen für Lehramtsstudierende und ausgebildete Lehrkräfte etabliert (u. a. Bruder, 2011; Gartmeier, 2018; Hertel, 2009).

Da Beratungskompetenz nicht nur die Ebene des Wissens, sondern auch die des Handelns umfasst (vgl. z. B. Hertel, 2009), beinhalten die entwickelten Interventionsmaßnahmen nicht nur Lerngelegenheiten, um wissenschaftlich fundiertes Wissen zu erwerben, sondern in der Regel auch praktische Ausbildungsanteile, die erste eigene Versuche des Beratungshandelns ermöglichen (z. B. Drechsel et al., 2020). Ein wichtiges Ziel der universitären Lehrkräftebildung ist es dabei, die Selbstwirksamkeit der angehenden Lehrkräfte in diesem Tätigkeitsfeld 
zu steigern und so handlungsbezogene Beratungskompetenz anzubahnen. Über die Wirksamkeit von Interventionen und deren Bestandteile ist bislang jedoch nur wenig bekannt. Insbesondere bleibt die Frage offen, inwieweit sich die theoretischen und praktischen Anteile der Qualifizierungsmaßnahmen auf den Erwerb von Beratungskompetenz von Lehramtsstudierenden auswirken. An dieses Forschungsdesiderat schließt der vorliegende Beitrag an und untersucht die Veränderung der beratungsbezogenen Selbstwirksamkeit und selbsteingeschätzten Beratungskompetenzen von Lehramtsstudierenden im Verlauf einer Intervention, die in Theorie- und Praxisphase unterteilt ist. Erwartungsgemäß zeigte sich im Kontroll- und Interventionsgruppenvergleich nach der Intervention (unter Kontrolle des Prätests) eine signifikante Überlegenheit der Interventionsgruppe hinsichtlich der Selbstwirksamkeitserwartung. In der Kontrollgruppe blieb die eigene Wirksamkeitsüberzeugung stabil.

Ausgehend von der theoretischen Annahme, dass direkte persönliche Erfahrungen den größten Einfluss auf die eigene Wirksamkeitsüberzeugung haben (Schwarzer \& Jerusalem, 2010; Tschannen-Morane et al., 1998) wurde angenommen, dass der praktische Interventionsteil entscheidend zur Förderung der Selbstwirksamkeitsüberzeugung beiträgt. Unter näherer Betrachtung der Wirkungsweise wurde deutlich, dass die Veränderung erst durch den Praxisteil erreicht werden konnte, während keine Veränderung nach dem Theorieteil beobachtet wurde. Das Ergebnis bestätigt somit die Annahme, dass hybride Lehrangebote Selbstwirksamkeit steigern können (z. B. Aich, 2011; Gartmeier, 2018; Gerich, 2017; Mergler \& Tangen, 2010; Lubitz, 2007), und spezifiziert diese, da sich v.a. der praktische Interventionsteil als wirksam erwies. Zusätzlich konnte gezeigt werden, dass auch konstruierte Praxisphasen im universitären Kontext Selbstwirksamkeit fördern (nicht nur Praktika im schulischen Kontext, z. B. Woolfolk Hoy \& Burke Spero, 2005; Swan et al., 2011), was besonders für den Bereich der Beratung gewinnbringend erscheint. Bei der Interpretation der Ergebnisse ist zu berücksichtigen, dass alle Studierenden zunächst den theoretischen Teil der Intervention besuchten, da dessen Inhalte als Voraussetzung für die praktischen Übungen notwendig sind. Inwiefern die Steigerung der Selbstwirksamkeit demzufolge auf den praktischen Teil oder die Kombination von Theorie und Praxis zurückzuführen ist, kann nicht beurteilt werden, ist jedoch ein guter Ansatzpunkt für weiterführende Forschung.

Untersucht wurde auch die Entwicklung der selbsteingeschätzten beratungsbezogenen Kompetenzen im Verlauf der Intervention. Die Ergebnisse zeigen eine Steigerung mit sehr hoher Effektstärke. In Bezug auf die Wirkung von Theorie und Praxis zeigten sich signifikante Veränderungen zu allen Messzeitpunkten. Erklärt werden kann das Befundmuster möglicherweise ausgehend von der Annahme, dass die subjektive Bewertung der eigenen Kompetenzen erst durch die Erfahrung der Anforderungssituation in einer veränderten Selbstwirksamkeit mündet (Tschannen-Morane et al., 1998).

In der vorliegenden Studie konnte, wie bereits bei Lubitz (2007) und Hertel (2009), eine Verbesserung der Selbstwirksamkeitserwartung durch die gezielte Interventionsmaßnahme gezeigt werden. Eine differenziertere Erkenntnis aus der vorliegenden Untersuchung ist, dass praktische Übungseinheiten mit Anregung zur Selbstreflexion einen signifikanten Effekt auf die beratungsbezogene Selbstwirksamkeit haben und deshalb essentielle Bestandteile von Qualifizierungsmaßnahmen sein sollten. Die universitäre Lehrkräfteausbildung steht vor der Herausforderung, solche praktischen Erfahrungen auch bei hohen bzw. steigenden Studierendenzahlen im Lehramt zu ermöglichen und bestenfalls alle Lehramtsstudierenden im Rahmen ihres regulären Studiums zu qualifizieren. Basierend auf den Ergebnissen der vorliegenden Studie scheinen Peer-Education-Konzepte ein geeigneter Ansatz zu sein, um dieser Herausforderung zu begegnen (vgl. Drechsel et al., 2020; Fricke et al., 2020)

Neben diesen Erkenntnissen hinsichtlich der Wirkungsweise von Theorie und Praxis auf 
die Selbstwirksamkeitsentwicklung sollte limitierend darauf hingewiesen werden, dass die Untersuchung der Wirksamkeit durch das quasi-experimentelle Design der Studie nur eingeschränkt möglich ist. Zudem sind die Aussagen über die Wirkung von Theorie und Praxis limitiert, da die Kontrollgruppe nur zu zwei Messzeitpunkten befragt wurde. Begrenzend ist ebenfalls festzuhalten, dass die praktischen Übungen der Qualifizierungsmaßnahme im universitären Kontext konstruiert und nicht authentisch im schulischen Kontext durchgeführt wurden. Der objektive Wissenszuwachs durch die Intervention wurde in vorliegendem Beitrag nicht untersucht (vgl. Weinhardt, 2014) und anhand der Selbsteinschätzungen wurde nur eine begrenzte Facette der Kompetenzen erfasst (König et al., 2012). Die Erfassung der selbsteingeschätzten Kompetenzen beruhte dabei auf einem eindimensionalen Instrument, obgleich vorherige Forschungsbefunde Kompetenzen mehrdimensional konzeptualisieren konnten (z. B. Gerich, 2017; Gartmeier, 2018; Hertel, 2009). Diese Diskrepanz könnte auf die Zielgruppe (Studierende) sowie den Fokus auf die Seminarinhalte zurückzuführen sein.

Zusammenfassend kann angesichts der mit diesem Beitrag vorliegenden Befundlage angenommen werden, dass gezielte Interventionen zur Förderung von Beratungskompetenz bei Lehramtsstudierenden mit theoretischen und praktischen Anteilen eine positive Auswirkung auf selbsteingeschätzte Kompetenzen haben können, und dass durch praktische Übungen auch im Bereich der Selbstwirksamkeitserwartung zum Thema Beratung im schulischen Kontext Progression erzielt werden kann.

In zukünftigen Studien könnte der Einbezug anderer Kompetenzfacetten, beispielsweise mit einem objektiven Wissenstest (vgl. Drechsel, 2020) oder durch performanzorientierte Simulationsverfahren (vgl. Aich, 2011; Gartmeier, 2018), weitere Erkenntnisse zur Wirkung von Theorie und Praxis zur Steigerung der Beratungskompetenzen in der Lehrkräftebildung liefern. Die Ergebnisse der vorliegenden Studie zeigen, dass praktische Übungen eine wichtige Rolle für die Entwicklung von Beratungskompetenzen spielen. Zukünftige Untersuchungen können hier anknüpfen und unterschiedliche praktische Erfahrungen im Beratungshandeln der Studierenden in ihrer Wirkungsweise überprüfen.

\section{Literatur}

Aich, G. (2011). Kompetente Lehrer. Ein Konzept zur Verbesserung der Konflikt-und Kommunikationsfähigkeit. Baltmannsweiler: Schneider.

Aich, G. (2015). Professionalisierung von Lehrenden im Eltern-Lehrer-Gespräch. Entwicklung und Evaluation eines Trainingsprogramms (Schul- und Unterrichtsforschung, Bd. 14, 2. unveränderte Auflage). Zugl.: Schwäbisch Gmünd, Pädagog. Hochschule., Diss., [2011]. Baltmannsweiler: Schneider.

Bach, A. (2013). Kompetenzentwicklung im Schulpraktikum: Ausmaß und zeitliche Stabilität von Lerneffekten hochschulischer Praxisphasen. Münster, u. a.: Waxmann Verlag.

Ball, D. L. \& Forzani, F. M. (2009). The work of teaching and the challenge for teacher education. Journal of Teacher Education, 60, 497-511.

Bandura, A. (1986). Social foundations of thought and action. A social cognitive theory (Prentice Hall series in social learning theory, 7 th print). Englewood Cliffs, N. J.: Prentice Hall.

Bandura, A. (2007). Self-efficacy. The exercise of control. Princeton, N. J.: Recording for the Blind \& Dyslexic.

Baumert, J. \& Kunter, M. (2006). Stichwort: Professionelle Kompetenz von Lehrkräften. Zeitschrift für Erziehungswissenschaft, 9, 469-520.

Baumert, J. \& Kunter, M. (2013). The COACTIV model of teachers' professional competence. In M. Kunter, J. Baumert, W. Blum, U. Klusmann, S. Krauss \& M. Neubrand (Hrsg.), Cognitive activation in the mathematics classroom and professional competence of teachers. Results from the COACTIV project (S. 25-48). New York: Springer.

Braun, E. \& Hannover, B. (2009). Zum Zusammenhang zwischen Lehr-Orientierung und Lehr-Gestaltung von Hochschuldozierenden und subjektivem Kompetenzzuwachs bei Studierenden. In M. A. Meyer, M. Prenzel \& S. Hellekamps (Hrsg.), Perspektiven der Didaktik (S. 476-491). Wiesbaden: VS Verlag für Sozialwissenschaften.

Bruder, S. (2011). Lernberatung in der Schule. Ein zentraler Bereich professionellen Lehrerhandelns. Dissertation, Technische Universität Darmstadt. Verfügbar unter http://tuprints.ulb.tu-darmstadt.de/2432/.

Bruder, S., Hertel, S., Gerich, M. \& Schmitz, B. (2014) Lehrer als Berater. In E. Terhart, H. Bennewitz \& M. Rothland (Hrsg.), Handbuch der Forschung zum Lehrerberuf (2. überarb. Aufl., S. 905-919). Münster, New York: Waxmann.

Bruder, S., Hertel, S. \& Schmitz, B. (2010). Lehrer als Berater. In E. Terhart, H. Bennewitz \& M. Rothland (Hrsg.), Handbuch der Forschung zum Lehrerberuf (S. 718-730). Münster: Waxmann. 
Cohen, J., Cohen, P., West, S. G. \& Aiken, L. S. (2003). Applied multiple regression/correlation analysis for the behavioral sciences (3rd edition). Mahwah, N.J. Lawrence Erlbaum.

Cramer, C. (2010). Kompetenzerwartungen Lehramtsstudierender. Grenzen und Perspektiven selbsteingeschätzter Kompetenzen in der Lehrerbildungsforschung. In A. Gehrmann, U. Hericks \& M. Lüder (Hrsg.), Bildungsstandards und Kompetenzmodelle: Beiträge zu einer aktuellen Diskussion über Schule, Leh rerbildung und Unterricht (S. 85-97). Bad Heilbrunn: Klinkhardt.

Drechsel B., Sauer D., Paetsch J., Fricke J. \& Wolstein J. (2020). Beratungskompetenz von Lehramtsstudierenden im erziehungswissenschaftlichen Studium evidenzbasiert fördern - Das Bamberger Peer-Beratungstrai ning. In I. Gogolin, B. Hannover \& A. Scheunpflug (Hrsg.), Evidenzbasierung in der Lehrkräftebildung (Edition ZfE, 4, S. 193-216). Wiesbaden: Springer VS

Dotger, B. H., Dotger, S. C. \& Maher, M. J. (2010). From medicine to teaching. The evolution of the Simulated Interaction Model. Innovative Higher Education, 35, 129- 141 .

Dotger, B. H., Harris, S. H. \& Hansel, A. (2008). Emerging authenticity. The crafting of simulated parent-teacher candidate conferences. Teaching Education, 19, 335347.

Dusolt, H. (2008). Elternarbeit als Erziehungspartnerschaft (3. überarb. Auflage) Weinheim: Beltz Verlagsgruppe.

Freyaldenhoven, I. (2005). Schule in der Krise? - Psychologische Beratung als Antwort! Theoretische und praktische Hinweise für eine gelingende Schulberatung - lösungsorientiert und individuell. Stuttgart: ibidem-Verlag.

Fuller, C. \& Taylor, P. (2012). Therapie-Tools. Motivierende Gesprächsführung. Weinheim: Beltz.

Furr, S. R. \& Carroll, J.J. (2003). Critical incidents in student counselor development. Journal of Counseling \& Development, 81, 483-489.

Fricke, J., Bauer-Hägele, S., Horn, D., Grötzbach, D., Sauer, D., Paetsch, J., Drechsel, B. \& Wolstein, J. (2020): Peer-Learning in der Lehrer*innenbildung. Gemeinsam und auf Augenhöhe lernen. Journal für LehrerInnenbildung, 19(3), 16-29.

Gartmeier, M. (2018). Gespräche zwischen Lehrpersonen und Eltern. Herausforderungen und Strategien der Förderung kommunikativer Kompetenz. Wiesbaden: Springer Fachmedien Wiesbaden.

Gartmeier, M., Bauer, J., Fischer, M., Hoppe-Sevler, T., Karsten, G., Kiessling, C.... Prenzel, M. (2015). Fostering professional communication skills of future physicians and teachers: effects of e-learning with video cases and role-play. Instructional Science, 43, 443-462.

Gerich, M. (2017). Teachers' Counseling Competence in Parent-Teacher Talks. Modeling, Intervention, BehaviorBased Assessment. Wiesbaden: Springer Fachmedien Wiesbaden

Gerich, M., Trittel, M. \& Schmitz, B. (2017). Improving Prospective Teachers' Counseling Competence in $\mathrm{Pa}$ rent-Teacher Talks. Effects of Training and Feedback. Journal of Educational and Psychological Consultation, 27(2), 203-238.

Grewe, N. (2005). Gesprächsführung und Leitlinien der Beratung. In N. Grewe (Hrsg.), Praxishandbuch Beratung in der Schule, Grundlagen, Aufgaben und Fallbei spiele (S. 13-34). München, Neuwied: Luchterhand, Wolter Kluwe Deutschland GmbH.
Hertel, S. (2009). Beratungskompetenz von Lehrern. Kompetenzdiagnostik, Kompetenzförderung, Kompetenzmodellierung (Pädagogische Psychologie und Entwicklungspsychologie, Bd. 74). Dissertation. Münster, München u. a.: Waxmann.

Hertel, S. (2017). Elternberatung im Schulalltag: Was wissen wir aus der Forschung und welche Bedeutung haben die Befunde für die Elternarbeit an Schulen? In G. Aich, C. Kuboth, M. Gartmeier \& D. Sauer (Hrsg.), Kommunikation und Kooperation mit Eltern (Pädagogik, 1. Auflage, S. 47-61). Weinheim: Beltz.

Hertel, S. \& Schmitz, B. (2010). Lehrer als Berater in Schule und Unterricht (Pädagogische Psychologie, 1. Aufl.). Stuttgart: Kohlhammer.

Jennings, L., Goh, M., Skovholt, T. M., Hanson, M. \& Banerjee-Stevens, D. (2003). Multiple factors in the development of the expert counselor and therapist. Journal of Career Development, 30, 59-72.

Klingebiel, F., Mähler, M. \& Kuhn, H. P. (2020). Was bleibt? Die Entwicklung der subjektiven Kompetenzeinschätzung im Schulpraktikum und darüber hinaus. In I. Ulrich \& A. Gröschner (Hrsg.). Praxissemester im Lehramtsstudium in Deutschland: Wirkung auf Studierende (S. 179-208). Wiesbaden: Springer VS.

König, J., Kaiser, G. \& Felbrich, A. (2012). Spiegelt sich pädagogisches Wissen in den Kompetenzselbsteinschätzungen angehender Lehrkräfte? Zum Zusammenhang von Wissen und Überzeugungen am Ende der Lehrerausbildung. Zeitschrift für Pädagogik, 58, 476-491.

Korthagen, F. A. J. (2010). The Relationship Between Theory and Practice in Teacher Education. In P. Peterson, E. Baker \& B. McGaw (Hrsg.), International Encyclopedia of Education (Volume 7, S. 669-675). Oxford: Elsevier.

Kücholl, D., Lazarides, R. \& Westfahl, A. (2020). Selbstwirksamkeitserwartungen in Beratung von Schülerinnen und Schülern: Welche Rolle spielen individuelle Eingangsvoraussetzungen angehender Lehrkräfte? Beiträge zur Lehrerinnen- und Lehrerbildung, 38, $150-166$.

Kücholl, D., Lazarides, R. C., Westphal, A. Lohse-Bossenz, H. (2018). Skala zur Erfassung der Beratungskompetenz im Lehramtsstudium. Potsdamer Beiträge zur Lehrerbildung und Bildungsforschung, 29-42.

Kücholl, D., Westphal, A., Lazarides, R. \& Gronostaj, A (2019). Beanspruchungsfolgen Lehramtsstudierender im Praxissemester. Zeitschrift für Erziehungswissenschaft, 22, 945-966.

Kultusministerkonferenz (2004). Standards für Lehrerbildung. Beschluss der Kultusministerkonferenz vom 16.12.2004. Zugriff am 17.11.2018 unter http://www.kmk.org/ fileadmin/Dateien/veroeffentlichungen_beschluesse/ 2004/2004_12_16-Standards-Lehrerbildung.pdf

Kunina-Habenicht, O., Lohse-Bossenz, H., Kunter, M. Dicke, T., Förster, D., Gößling, J. ... Terhart, E. (2012). Welche bildungswissenschaftlichen Inhalte sind wichtig in der Lehrerbildung? Zeitschrift für Erziehungswissenschaft, 15, 649-682.

Kunter, M. \& Pohlmann, B. (2015). Lehrer. In E. Wild \& J. Möller (Hrsg.), Einführung in die Pädagogische Psychologie (S. 261-281). Berlin: Springer.

Lambert, M. J., Whipple, J. L., Vermeersch, D. A., Smart, D. W., Hawkins, E. J., Nielsen, S. L. \& Goates, M. (2002). Enhancing psychotherapy outcomes via providing feedback on client progress: A replication. Clin. Psychol. Psychother. 9, 91-103. 
Lent, R. W., Larkin, K. C. \& Brown, S. D. (1986). SelfEfficacy in the Prediction of Academic Performance and Perceived Career Options. Journal of Counseling Psychology, 33, 265-269.

Lubitz, I. (2007). Soziale Kompetenzen im Lehrerberuf. Konzeption und Evaluation eines Kurztrainings in der Lehrerausbildung (Schriften zur pädagogischen Psychologie, Bd. 27). Zugl.: Braunschweig, Techn. Univ. Diss., 2006 u.d.T.: Lubitz, Ilona: Konzeption und Evaluation eines Kurztrainings sozialer Kompetenzen in der universitären Lehrerausbildung. Hamburg: Kovač. Verfügbar unter http://www.verlagdrkovac.de/ 978-3-8300-2777-5.htm

McLeod, J. (2013). An Introduction to Counselling (5. Ed.). New York: Open University Press.

Mergler, A. G. \& Tangen, D. (2010). Using microteaching to enhance teacher efficacy in pre-service teachers. Teaching Education, 21, 199-210.

Meyer, J. M. (2014). Counseling Self-Efficacy: On-Campus and Distance Education Students. Rehabilitation Counseling Bulletin, 58, 165-172.

Nußbeck, S. (2006). Einführung in die Beratungspsychologie (2. Aufl.). Stuttgart: UTB.

Pajares, F. \& Kranzler, J. (1995). Self-efficacy beliefs and general mental ability in mathematical problem-solving. Contemporary Educational Psychology, 20, 426-443.

Renkl, A. (2014). Wissenserwerb. In E. Wild \& J. Möller (Hrsg.), Pädagogische Psychologie (S. 3-26). Berlin: Springer.

Rogers, C. R. (1951). Client-Centered Therapy. Boston: Houghton Mifflin.

Rønnestad, M. H. \& Skovholt, T. M. (2003). The journey of the counselor and therapist: Research findings and perspectives on professional development. Journal of Career Development, 30, 5-44.

Rothland, M. (2013). Belastung und Beanspruchung im Lehrerberuf. Modelle, Befunde, Interventionen. Wiesbaden: Springer VS.

Sauer, D. (2015). Wie Beraten Lehrkräfte Eltern? Eine Qualitativ-Rekonstruktive Studie Zur Beratungsaufgabe Von Lehrkräften (ZBBS-Buchreihe). Leverkusen-Opladen Barbara Budrich-Esser.

Sauer, D. (2017). Beratungs-, Rückmelde- oder Konfliktgespräch? - Von der Bedeutung verschiedener Gesprächsanlässe im Lehrer-Eltern-Gespräch. In G. Aich, C. Kuboth, M. Gartmeier, D. Sauer (Hrsg.), Kommu nikation und Kooperation mit Eltern (S. 101-111). Weinheim, Basel: Beltz.

Schipper, T. M., Goei, S. L., de Vries, S. \& van Veen, K. (2018). Developing teachers' self-efficacy and adaptive teaching behaviour through lesson study. International Journal of Educational Research, 88, 109-120.

Schlippe, A. \& Schweitzer, J. (2010). Lehrbuch der systemischen Therapie und Beratung. Göttingen u. a.: Vandenhoeck \& Ruprecht.

Schnebel, S. \& Jürgens, E. (2012). Professionell beraten Beratungskompetenz in der Schule (Pädagogik 2013, 2. Aufl.). Weinheim: Beltz.

Schüle, C., Besa, K.-S., Schriek, J. \& Arnold, K.-H. (2017). Die Veränderung der Lehrerselbstwirksamkeitsüberzeugung in Schulpraktika. Zeitschrift für Bildungsforschung, 7, 34-40.

Schulte, K., Bögeholz, S. \& Watermann, R. (2008). Selbstwirksamkeitserwartung und Pädagogisches Professions wissen im Verlauf des Lehramtsstudiums. Dissertation, Georg-August-Universität, Göttingen.
Schwarzer, C. \& Posse, N. (2005). Beratung im Handlungsfeld Schule. Pädagogische Rundschau, 59(2), 139-151.

Schwarzer, R. \& Jerusalem, M. (2010). Das Konzept de Selbstwirksamkeit. In D. Hopf \& M. Jerusalem (Hrsg.), Selbstwirksamkeit und Motivationsprozesse in Bildungsinstitutionen (Zeitschrift für Pädagogik Beiheft, Bd. 44, Unveränd. Nachdr. der letzten Aufl., S. 28-53). Weinheim: Beltz.

Siegel, R. G., Galassi, J. P. \& Ware, W. B. (1985). A Comparison of Two Models for Predicting Mathematics Performance: Social Learning Versus Math AptitudeAnxiety. Journal of Counseling Psychology, 32, 531- 538. Strasser, J. \& Gruber, H. (2003). Kompetenzerwerb in der Beratung: Eine kritische Analyse des Forschungsstandes. Psychologie in Erziehung und Unterricht, 50, 381-399. Suhlmann, L.S. (1986). Those who understand: Knowledge growth in teaching. Educational Researcher, 15, 4-14.

Summers, J., Davis, H.A. \& Woolfolk Hoy, A. (2017). The effects of teachers' efficacy beliefs on students' perceptions of teacher relationship quality. Learning and Individual Differences, 53, 17-25.

Swan, B. G., Wolf, K. J. \& Cano, J. (2011). Changes in teacher self-efficacy from the student teaching experience through the third year of teaching. Journal of Agricultural Education, 52(2), 128-139.

Tang, M., Addisson, K. D., LaSure-Bryant, D., Norman, R. O'Connell, W., Stewart-Sicking, J. A. (2004). Factors That Influence Self-Efficacy of Counseling Students: An Exploratory Study. Counselor Education and Supervision, 44(1), 70-80

Thiel, H.-U. (2003). Phasen des Beratungsprozesses. In C. Krause, B. Fittkau, R. Fuhr \& H.-U. Thiel (Hrsg.). Pädagogische Beratung - Grundlagen und Praxisanwendung (S. 73-84). Paderborn u. a.: UTB.

Tschannen-Morane, M., Woolfolk Hoy, A. \& Hoy, W. K. (1998). Teacher Efficacy: Its Meaning and Measure. Review of Educational Research, 68, 202-248.

Watts, M., \& Lawson, M. (2009). Using a meta-analysis activity to make critical reflection explicit in teacher education. Teaching and Teacher Education, 25, 609616.

Weinhardt, M. (2014). Kompetenzentwicklung in der psychosozialen Beratung am Beispiel von Studierenden der Erziehungswissenschaft. In P. Bauer \& M. Weinhardt (Hrsg.), Perspektiven sozialpädagogischer Beratung. Empirische Befunde und aktuelle Entwicklungen (Edition Soziale Arbeit, S. 214-231). Weinheim: Beltz Juventa.

Weß, R., Priemer, B., Weusmann, B., Ludwig, T., Sorge, S. \& Neumann, I. (2020). Der Verlauf von lehrbezogenen Selbstwirksamkeitserwartungen angehender MINT-Lehrkräfte im Studium. Zeitschrift für Pädagogische Psychologie, 5, 1-18.

Wild, E. (2003). Lernen lernen. Wege einer Förderung der Bereitschaft und Fähigkeit in sozialpädagogische und psychosoziale Beratungsansätze. Unterrichtswissenschaft, 31, 2-5.

Wild, E. \& Lütje-Klose, B. (2017). Schulische Elternarbeit als essenzielles Gestaltungsmoment inklusiver Beschulung. In B. Lütje-Klose, S. Miller, S. Schwab, B. Streese \& Österreichische Gesellschaft für Forschung und Entwicklung im Bildungswesen (ÖFEB) (Hrsg.) Inklusion: Profile für die Schul-und Unterrichtsentwick lung in Deutschland, Österreich und der Schweiz. Theoretische Grundlagen, empirische Befunde, Praxisbeispiele (1. Aufl., S. 129-139). Münster: Waxmann Verlag. 
Wildt, B. (2004). Beratung. In S. Blömeke (Hrsg.), Handbuch Lehrerbildung (S. 519-530). Bad Heilbrunn/Obb.: Klinkhardt.

Woolfolk Hoy, A. \& Burke Spero, R. (2005). Changes in teacher efficacy during the early years of teaching: A comparison of four measures. Teaching and Teacher Education, 21, 343-356.

Zee, M. \& Koomen, H. M. (2016). Teacher Self-Efficacy and Its Effects on Classroom Processes, Student Academic Adjustment, and Teacher Well-Being: A Synthesis of 40 Years of Research. Review of Educational Research, 86, 981-1015.

Zimmerman, B. J. (2000). Self-Efficacy: an essential motive to learn. Contemporary Educational Psychology, 52, $82-91$.
Zimmerman, B. J. \& Kitsantas, A. (1997). Developmental Phases in Self-Regulation: Shifting From Process Goals to Outcome Goals. Contemporary Educational Psycho$\log y, 89,29-36$.

\section{Anne Schlosser}

Prof. Dr. Jennifer Paetsch

PD Dr. Daniela Sauer

Luitpoldstraße 19

96047 Bamberg

E-Mail: Anne.Schlosser@uni-bamberg.de Jennifer.Paetsch@uni-bamberg.de Beratung.Wege@uni-bamberg.de 